\title{
Acinetobacter baumannii Biofilm Formation and Its Role in Disease Pathogenesis: A Review
}

\author{
Alemu Gedefie (D) \\ Wondmagegn Demsis' \\ Melaku Ashagrie (iD) \\ Yeshimebet Kassa' \\ Melkam Tesfaye' \\ Mihret Tilahun (D)' \\ Habtye Bisetegn (1D) \\ Zenawork Sahle (iD) ${ }^{2}$ \\ 'Department of Medical Laboratory \\ Sciences, College of Medicine and Health \\ Science, Wollo University, Dessie, \\ Ethiopia; ${ }^{2}$ Department of Medical \\ Laboratory Sciences, Debre Birhan \\ Health Science College, Debre Birhan, \\ Ethiopia
}

Correspondence: Alemu Gedefie Email alemugedefie@gmail.com

\begin{abstract}
Acinetobacter species, particularly Acinetobacter baumannii, is the first pathogen on the critical priority list of pathogens for novel antibiotics to become a "red-alert" human pathogen. Acinetobacter baumannii is an emerging global antibiotic-resistant gram-negative bacteria that most typically causes biofilm-associated infections such as ventilator-associated pneumonia and catheter-related infection, both of which are resistant to antibiotic therapy. A. baumannii's capacity to develop antibiotic resistance mechanisms allows the organism to thrive in hospital settings, facilitating the global spread of multidrug-resistant strains. Although Acinetobacter infections are quickly expanding throughout hospital environments around the world, the highest concentration of infections occurs in intensive care units (ICUs). Biofilms are populations of bacteria on biotic or abiotic surfaces that are encased in the extracellular matrix and play a crucial role in pathogenesis, making treatment options more difficult. Even though a variety of biological and environmental elements are involved in the production of $A$. baumannii biofilms, glucose is the most important component. Biofilm-mediated A. baumannii infections are the most common type of A. baumannii infection associated with medical equipment, and they are extremely difficult to treat. As a result, health care workers (HCWs) should focus on infection prevention and safety actions to avoid A. baumannii biofilm-related infections caused by medical devices, and they should be very selective when using treatments in combination with anti-biofilms. Therefore, this review discusses biofilm formation in $A$. baumannii, its role in disease pathogenesis, and its antimicrobial resistance mechanism.
\end{abstract}

Keywords: biofilm, Acinetobacter, A. baumannii, pathogenesis, antibiotics resistance

\section{Introduction}

Acinetobacter baumannii is the member of the genus Acinetobacter and the family Moraxellaceae of the Eubacteria class Proteobacteria. ${ }^{1}$ Acinetobacter baumannii is non-motile, non-fastidious, non-fermentative, catalase-positive, oxidative-negative Gram-negative coccobacilli. ${ }^{2}$ Acinetobacter baumannii is an ESKAPE pathogen (Enterococcus faecium, Staphylococcus aureus, Klebsiella pneumoniae, Acinetobacter baumannii, Pseudomonas aeruginosa, and Enterobacter species) ${ }^{3}$ associated with hospital-acquired antibiotic-resistant infections. Of all hospitalacquired infections caused by Gram-negative; A. baumannii is responsible for 2$10 \%$ of infections. ${ }^{4}$ With invasive operations and frequent antibiotic use as well as immunocompromised hosts, A. baumannii has emerged as a major nosocomial pathogen. The pathogen's versatile genetic machinery allows it to rapidly generate resistance factors, as well as a remarkable ability to tolerate harsh environmental circumstances, making it endemic in hospital settings. ${ }^{2}$ 
A. baumannii is a low-grade pathogen that can be found in a variety of habitats, including soil, water, and food, and is frequently isolated from medical devices. ${ }^{5}$ As a result of colonization and living on much medical equipment, it causes severe infections in immune-compromised people. ${ }^{6}$ According to evidence meningitis, wound, pneumonia (hospital and community-acquired), bacteremia, burn, endocarditis, urinary tract infections (UTI) as well as skin and soft tissue infections are the most prevalent Acinetobacter-associated nosocomial illnesses. ${ }^{5,7}$ Community-acquired pneumonia is more severe than nosocomial pneumonia; it is usually fulminant, and fatality rates can reach $60 \%{ }^{8}$ This human pathogen is also responsible for ventilator-associated pneumonia and bloodstream infections, both of which have high death rates of up to $35 \%{ }^{9}$ A. baumannii infections are more common in men and are linked to advanced age, behavioral factors like alcoholism and excessive smoking as well as chronic disease comorbidities like diabetes, renal disease, and chronic obstructive pulmonary disease (COPD). ${ }^{8}$

The most important virulence factors identified by genomic and phenotypic investigations are outer membrane porins, phospholipases, capsular polysaccharides, lipopolysaccharides (LPS), proteases, iron-chelating systems, and protein secretion systems. ${ }^{2,7,10}$ A. baumannii infections are often associated with multidrug resistance; Carbapenem and Colistin resistance has been observed. Surprisingly, antibiotic-resistant bacteria have lately surfaced. ${ }^{9}$ Modifications of target sites, permeability deficiencies, multidrug efflux pumps, and enzymatic drug degradation, for example, $\beta$-lactamases and aminoglycoside-modifying enzymes, are all linked to A. baumannii resistance mechanisms. ${ }^{2,11,12}$ The fast spread of multidrug resistance in A. baumannii is currently posing a severe threat to public health. The ability of A. baumannii to colonize and produce biofilm on biotic and abiotic surfaces contributes to chronic and persistent infections, antibiotic resistance, as well as survival in hospital environments and transfer. ${ }^{13}$ Hence, the biofilm matrix that surrounds bacteria permits germs to withstand extreme circumstances and resist treatments of antibiotics. As a result, medications now available for treating A. baumannii biofilmassociated infections are ineffective. ${ }^{14}$

Biofilms are communities of microorganisms which are adhering to biotic and/or abiotic surface encased by extracellular polymeric substance (EPS) matrix and are physiologically different from planktonic (free floating) bacteria. ${ }^{15}$ Biofilm-encased cells have limited metabolic activity and are shielded by the extracellularly produced matrix, making them more resistant to antibiotics and innate immune components of the host. ${ }^{16}$ A. baumannii frequently causes biofilm-related infections, particularly ventilator-associated pneumonia and catheter-related infection, which can be exceedingly resistant to antibiotic therapy, offering a severe challenge to the clinical management of A. baumannii-related biofilm infections. ${ }^{5,17}$ A. baumannii biofilms have become one of the most serious global issues due to the rapid spread of medical device-associated infections and antibiotic resistance. ${ }^{9,13}$ Despite these problems causes, the data about A. baumannii biofilms in Africa are still limited. Hence, understanding the magnitude of $A$. baumannii biofilm formation; its role in pathogenesis and antimicrobial resistance is important for limiting medical device-associated infections. Therefore, the objective of this review is to discuss A. baumannii biofilm formation and its role in disease pathogenesis.

\section{Discussion}

\section{Magnitude of Acinetobacter baumannii Biofilm Formation}

The relationship between biofilm development and multidrug resistance was investigated in a study. There were 156 confirmed A. baumannii isolates, $10.26 \%$ of which produced biofilms on Congo red agar. However, in test tube, conventional microtiter plate, and modified microtiter plate assays, the percentage of bacterial isolates with positive biofilm was $48.72 \%, 66.66 \%$, and $73.72 \%$, respectively. ${ }^{18}$ The effectiveness of isolated bacteriophage against biofilm-embedded Colistin-Resistant Acinetobacter baumannii isolates was investigated in another investigation. It was discovered that $20 \%$ of isolates formed a mild biofilm, $40 \%$ created an intermediate biofilm, and $40 \%$ produced a strong biofilm. ${ }^{19}$ Another similar study also revealed that $80-98 \%$ of strains were biofilm producers. $^{20}$ Acinetobacter baumannii isolates from 92 unrelated strains were tested for biofilm production using a microtiter plate assay in a multicenter cohort study, and $63 \%$ of the isolates produced biofilm. ${ }^{21}$

Surprisingly, in a study done on $100 \mathrm{~A}$. baumannii clinical isolates from immunocompromised hospitalized patients in intensive care unit (ICU); all isolates had the ability to form a biofilm, and 58\% of isolates showing high biofilm-forming ability. Molecular typing of biofilmrelated genes based on REP-PCR showed that the 
distribution of csuE, pgaB, epsA and ptk, bfmS, and ompA genes were $100 \%, 98 \%, 95 \%, 92 \%$, and $81 \%$ respectively. Nearly total $(98 \%)$ of isolates simultaneously carried more than 4 biofilm-related genes. ${ }^{22}$ In another similar study, seventy-five biofilm-producing multidrug-resistant Acinetobacter species were identified in a study by the microtiter plate method. Of these 75 isolates 12 (16\%), 9 $(12 \%), 30(40 \%)$, and $24(32 \%)$ respectively were weak biofilm producers, moderate biofilm producers, strong biofilm producers, and non-biofilm producers. This study found a clear relationship between Acinetobacter isolates' propensity to form biofilm and the development of biofilm and multiple antibiotic resistance. ${ }^{23}$

Moreover, among 125 clinical samples from skin infections in patients of Baghdad, 18 (14.4\%) were identified as A. baumannii. Different findings were observed across the different techniques used. On the Tissue Culture Plate method 1, 15, and 2 isolates respectively were identified as weak, moderate, and strong biofilm producers among 18 isolates. Biofilm production was not detected in any of these isolates in Congo red agar but on Tube Method 7, 5 and 6 isolates respectively were weak, moderate, and strong biofilm producers. ${ }^{24}$ Another study also revealed that 34 isolates $(62 \%)$ were biofilm producers, with only 11 having the blaPER-1 gene, two of which were significant biofilm producers and the rest were weakly adherent isolates. $^{25}$

The molecular analysis and expression of the bap Gene in biofilm-forming A. baumannii were investigated. The genes ompA and csuE were found in all of the isolates, while bap and blaPER-1 were found in 43 (66\%) and 42 $(64 \%)$ of the isolates with strong and moderate biofilm activity ( $p$ 0.05), respectively. Furthermore, strong, moderate, weak, and no biofilm activities were found in 23 (35.4\%), $18(27.7 \%), 13(20 \%)$, and $11(16.9 \%)$ of the isolates, respectively. In the presence of low iron concentrations, overexpression of bap influences biofilm development, according to the findings of this study. ${ }^{26}$

\section{Clinical Significances}

Acinetobacter baumannii is a common bacterium that causes nosocomial or hospital-acquired illnesses. Due to the rise of multidrug-resistant strains and high morbidity and mortality, A. baumannii has been added to the World Health Organization's (WHO) drug-resistant bacteria and antimicrobial resistance research priority list. $^{27}$ A. baumannii is causing a wide range of infections related to medical devices, eg, vascular catheters, cerebrospinal fluid shunts, or Foley catheters ${ }^{21}$ as well as a postoperative infection like urinary tract infections (UTI) in hospitalized patients. $^{4}$ Moreover, A. baumannii causes respiratory tract infections (RTI), meningitis, UTI, wound infections, endocarditis, and bacteremia all of which involve the formation of biofilm. ${ }^{4,28}$ Antibiotic resistance in biofilms is thought to be 1000 times higher than in planktonic organisms. Hence, the high prevalence of $A$. baumannii infections is biofilm mediated which poses a global health concern. ${ }^{29}$

\section{Stages of Biofilm Development}

Biofilms are bacteria that have accumulated in an extracellular polymeric material matrix made up of polysaccharides, lipids, proteins, and nucleic acids. Biofilm development is a complicated process in which microorganism cells transform their growth mode from planktonic to sessile mode, and it is influenced by a variety of environmental conditions such as surface porosity, fluids flow, and availability of nutrients. ${ }^{30}$

The common steps in the development of biofilm are initial contact/attachment to the biotic and/or abiotic surface, micro-colony formation, maturation and formation of the architecture of the biofilm, and lastly detachment/dispersion of the biofilm, which are all controlled by quorum sensing (QS). ${ }^{30}$ Biofilm formation begins with the planktonic cell adhering to an abiotic or abiotic surface. A reversible connection follows adhesion cells. Microorganisms adhere more quickly to hydrophobic, nonpolar surfaces like Teflon and other plastics than to hydrophilic surfaces like stainless steel, implying that some sort of hydrophobic interaction occurs, allowing the cells to overcome the repulsive forces. ${ }^{31}$ After the attachment is stable; the EPS matrix fixes the initial adhesion then multiplication of microbial cells starts and the basic structural unit of the biofilm (ie, micro-colony) formation has occurred. The closeness of cells within the microcolony or across micro-colonies plays a significant role in the interchange of the substrate, as well as distribution of major metabolic products and excretion of metabolic end-products. $^{32}$

Biofilm maturation occurs when the irreversibly attached cells develop a more organized and complex structure and shape that is dependent on the source of nutrients. Microbial cells communicate with one another via a cell-to-cell signaling mechanism known as quorum sensing to control their population density using signaling molecules known as auto-inducers. ${ }^{33}$ Quorum sensing also aids in the transmission of favorable mutations in the 
biofilm colony, improves access to resources, and contributes to antibiotic tolerance. In this stage, the matrix also produces interstitial spaces (channels) that are filled with water and act as a circulatory system. ${ }^{34}$ Finally, the sessile form converts into motile form; ie, dispersion of biofilm occurred. Detachment/dispersion of biofilm is initiated by oxygen or nutrient starvation and dissolution of EPS by saccharolytic enzymes, allowing the bacteria surface to be released into a new location for colonization. ${ }^{32}$

\section{Biofilm in Disease Pathogenesis}

Infections associated with biofilms are first restricted to a specific location before detaching over a while. The detachable biofilms may then cause infections in the bloodstream or urinary system, as well as restriction of blood flow. ${ }^{35}$ On the contrary, sessile cells or biofilms are resistant to harsh environmental conditions, antimicrobial agents as well as host immune systems than planktonic bacteria. As a result, removing biofilms from living hosts is quite challenging. ${ }^{36}$

The ability of $A$. baumannii to survive in harsh environmental condition, dormancy of bacterial cells deep in the biofilm, multiple antibiotic resistance mechanisms, prolonging survival on inanimate objects and resistant to environmental stress plays a role in its environmental survival, resulting in biofilms causing a wide range of subacute or chronic infections which are very challenging to eradicate. $^{37,38}$ A. baumannii has been designated as a "redalert" human pathogen due to its ability to acquire resistance to all currently available antimicrobial agents. ${ }^{39}$

Even though $A$. baumannii infections are becoming increasingly important in clinical practice and are a global health concern, relatively little is known about the factors influencing its pathogenesis. Pieces of evidence elucidated that outer membrane protein A (OmpA), phospholipids, extracellular polysaccharides, ${ }^{2,10,38-40}$ the $\mathrm{K} 1$ capsule, a siderophore-mediated iron-acquisition system, and phospholipases are virulence factors that have an important role in bacterial pathogenicity ${ }^{2,10,38}$ (Table 1).

Furthermore, the formation ability of biofilm (due to fimbriae and pili) is a significant factor that aggravates the disease process of $A$. baumannii infections. ${ }^{10,38,40}$ After initial attachment to abiotic surfaces, pili assembly and synthesis of the biofilm-associated protein (Bap) surfaceadhesion protein play a significant role in the initiation and maturation of biofilm. ${ }^{41}$ Biofilm increases $A$. baumannii adherence and long-term survival on both biotic and abiotic surfaces. Adhesion to abiotic surfaces such as medical

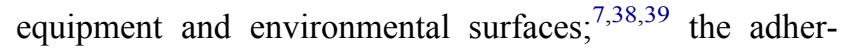
ence capacity of cells and the expression level of OMPs mRNAs are determinant virulence factors for A. baumannii pathogenesis. ${ }^{40}$ Additionally, OmpA contributes to biofilm formation on biotic surfaces like epithelial cells and mediates bacterial adherence to lung epithelial cells by interacting with fibronectin on the surface of cells and may induce apoptosis in human epithelial cells. ${ }^{40,42}$

\section{Factors Influencing Biofilm Formation}

A. baumannii is at least three times higher biofilm former at the solid-liquid interface than the other Acinetobacter species. Clinical strains can form stronger biofilms than environmental strains, and biofilm development on medically important surfaces affects their ability to resist nutrition availability stress, desiccation, and antimicrobial therapy. $^{28}$ The formation of biofilms is a complicated process that is influenced by a variety of cell properties. Cell surface, hydrophobicity, surface charge, adhesion proteins, and extracellular polymeric compounds all have an impact on the production of $A$. baumannii biofilms. ${ }^{28,62,63}$

Furthermore, the formation of the biofilm process of A. baumannii on abiotic surfaces has a positive correlation with multidrug resistance and with virulence factors expression such as the OmpA, the extracellular polysaccharide poly- $\beta_{-}{ }^{1,6}-\mathrm{N}$-acetyl glucosamine (PNAG), type I pili, Rec A, Bap, and the Omp CarO. On an animate surface, it needs chaperone-usher pili to produce biofilm. ${ }^{62}$ A. baumannii has biofilm-related virulence genes and proteins, which aid in its capacity to cling to biotic and abiotic surfaces and form biofilms. ${ }^{29}$

Environmental changes in physical and chemical settings have an impact on phenotypic characteristics and the expression of key functions. ${ }^{29}$ Several environmental elements influence biofilm growth in a highly regulated manner at each stage. Temperature, osmolarity, ferrous iron concentration, nutrients availability, quality of materials where biofilms are formed, light, and ambient acidic conditions are the most major environmental elements affecting biofilm formation. ${ }^{64}$ Glucose is also the key determinant factor in $A$. baumannii biofilm formation. Moreover, A. baumannii biofilm formation has been influenced by surface hydrophobicity and oxygen content. ${ }^{29}$

\section{Biofilm and Antimicrobial Resistance}

A. baumannii is inherently antibiotic-resistant. Due to the increasing usage of antibiotics, multidrug-resistant (MDR) and extensively drug-resistant (XDR) bacteria have 
Table I Summary of Known Acinetobacter baumannii Virulence Factors

\begin{tabular}{|c|c|c|}
\hline Virulence Factors (Genes) & Possible Role in the Pathogenesis & References \\
\hline I.Outer membrane protein $\mathrm{A}(\mathrm{OmpA})$ & $\begin{array}{l}\text { Epithelial cell adhesion and invasion, biofilm development, serum resistance, surface } \\
\text { motility, and induction of apoptosis in host cells or induces cytotoxicity }\end{array}$ & {$[40,42-45]$} \\
\hline 2.Biofilm-associated protein (Bap) & Biofilm formation and subsequent intercellular adhesion within the mature biofilm & [46] \\
\hline 3.Lipopolysaccharide(LPS) & $\begin{array}{l}\text { Host immune response evasion, resistance to cationic antimicrobial peptides, triggering } \\
\text { the host inflammatory response, reduces TLR4 signaling and desiccation survival }\end{array}$ & {$[47,48]$} \\
\hline 4.Penicillin-binding protein $7 / 8$ (pbpG) & Biosynthesis of peptidoglycan, cellular stability, and growth in serum & [49] \\
\hline 5.Outer membrane vesicles (OMV) & $\begin{array}{l}\text { Delivering virulence genes to host cells cytoplasm and transferring of genetic material } \\
\text { across bacterial cells }\end{array}$ & {$[50,5 \mathrm{I}]$} \\
\hline 6.Phospholipase D & Bacterial survival in vivo, serum resistance, and dissemination of bacteria & [52] \\
\hline $\begin{array}{l}\text { 7.Acinetobactin or Siderophore } \\
\text { mediated iron acquisition mechanism }\end{array}$ & Provides iron for the survival of the bacterium the host and induce cell death & {$[53,54]$} \\
\hline 8.Capsule & $\begin{array}{l}\text { Mediates cationic antimicrobial peptide resistance, as well as serum resistance and } \\
\text { in vivo survival }\end{array}$ & {$[10,55]$} \\
\hline 9.Phospholipase C & $\begin{array}{l}\text { Exhibiting hemolytic action against human red blood cells and assisting in the uptake of } \\
\text { iron }\end{array}$ & {$[56,57]$} \\
\hline $\begin{array}{l}\text { I0.Poly- } \beta-1-6-N-A c e t y l g l u c o s a m i n e \\
\text { (PNAG) }\end{array}$ & $\begin{array}{l}\text { Formation of biofilm, cell-cell adherence as well as protection against innate host } \\
\text { defenses }\end{array}$ & {$[39,58]$} \\
\hline $\begin{array}{l}\text { II.Two-component regulatory system, } \\
\text { BfmRS }\end{array}$ & $\begin{array}{l}\text { Csu pili chaperone-usher assembly system expression, biofilm formation, and cellular } \\
\text { morphology }\end{array}$ & [59] \\
\hline 12.Abal autoinducer synthase & Normal biofilm development & {$[60]$} \\
\hline $\begin{array}{l}\text { I3.CsuA/BABCDE chaperone usher pili } \\
\text { assembly system }\end{array}$ & Pilus assembly, biofilm formation on abiotic surfaces & {$[39,61]$} \\
\hline
\end{tabular}

evolved. As a result, the worldwide emergence of A. baumannii is becoming a critical problem. ${ }^{28}$ The emergence of MDR strains is a major cause of the high mortality rate. ${ }^{65}$ Even though the link between biofilm production and antibiotic resistance phenotypes is still debated. ${ }^{28}$ Pieces of evidence revealed that biofilm formation and multidrug resistance have a positive correlation. ${ }^{28,66}$ In comparison to imipenem and piperacillin, biofilm formers demonstrated increased resistance to ampicillin-sulbactam, amikacin, ciprofloxacin, and ceftazidime. Moreover, biofilm formation and multidrug-resistant have a statistical association. ${ }^{66}$ Another study found that clinical isolates of A. baumannii have a high proclivity for biofilm formation and that biofilms are associated with multiple drug resistance. This study also showed that the resistance pattern of antibiotics was also found to be higher among biofilm producers than among non-biofilm producers. ${ }^{25}$
A similar study was conducted on $A$. baumannii strains isolated from hospital-acquired infection for the relationship between biofilm production and drug susceptibility. Paradoxically, ceftazidime-sensitive strains formed less biofilm than ceftazidime resistant as well as tobramycin and amikacin sensitive strains produced more biofilm than strains showing resistance to these antibiotics. On the other hand, strong biofilm producers from ICUs are often more susceptible to antibiotics which are related to the fact that bacteria protected in biofilm do not need resistance mechanisms responsible for resistance by planktonic cells. ${ }^{20}$ Other studies showed that biofilm-forming isolates were less commonly resistant to imipenem and ciprofloxacin than non-biofilm-forming isolates. ${ }^{21}$ Similarly, meropenemresistant $A$. baumannii isolates had a lower ability to form biofilms than meropenem-susceptible isolates. ${ }^{67}$ 
Generally, Acinetobacter strains with multidrug resistance and biofilm production continue to pose a substantial hazard in the hospital setting, since they rapidly become resistant to routinely used routine medications, and their ability to produce biofilm is statistically significant with imipenem resistance. ${ }^{68}$ Enzyme-mediated neutralizations, the existence of persistent (non-dividing) cells, and the biofilm phenotype are all antimicrobial resistance mechanisms.

\section{Detection of Biofilm}

Different phenotypic techniques such as Qualitative biofilm production assays (ie, tube method and tissue culture plate method) and Quantitative biofilm production assays (ie, Congo red agar method) are used to detect biofilm formation A. baumannii. ${ }^{13,18,24}$ Additionally, Microscopic examination of the ability of $A$. baumannii strains to produce biofilms is seen using a scanning electron microscope (SEM), and detection of genes related to biofilm such as; ompA bap, blaPER-1and csuE genes are screened using Polymerase chain reaction (PCR). ${ }^{13}$ The modified microtiter plate assay for detecting $A$. baumannii biofilm is a sensitive, accurate, repeatable, and reliable quantitative assay. ${ }^{18,24}$

Tube Method and Tissue Culture Plate Methods need staining to visualize the presence of biofilm on different days using crystal violet dye. However, Congo red agar does not need stain and it can detect by culturing from another culture and producing a distinct color, which may be used to identify it. The biofilm producers become black colonies with a dry crystalline consistency and the nonproducers remain red or move. ${ }^{18,24}$ Biofilm production is graded into strong, moderate, and non/weak. Strong and moderate results are interpreted as positive biofilm production, while non/weak results are interpreted as negative biofilm production. ${ }^{24}$

\section{Therapeutics Options}

Despite this, early detection of biofilm infection is challenging at the moment, and the majority of clinical biofilm infections are mature biofilms that are difficult to eliminate with antibiotics. Because nascent biofilm can be eliminated more easily than matured biofilm, early and strong antibiotic therapies are indicated for biofilm infections. ${ }^{69}$

Because of the persistent nature of biofilm-associated A. baumannii infections, therapy can be difficult. However, because biofilm formation increases antibiotic resistance, combination therapy may be beneficial in treating biofilm-related infections. ${ }^{29}$ Therefore, the treatment of biofilm consists of a selection of antibiotics as sensitive and well penetrating. ${ }^{70}$ Well-established interdisciplinary teamwork is required for the effective treatment of biofilm infections. As a result, contaminated foreign bodies removal, use of biofilm-active, sensitive, and wellpenetrating antimicrobials, use of anti-quorum sensing or biofilm dispersal agents and systemic or topical antibiotic administration in high doses and combinations can be used for efficient treatment of biofilm-related infections. ${ }^{70,71}$

\section{Prevention and Control}

Biofilm infections induced by A. baumannii are notoriously difficult to treat. However, preventing these organisms from forming biofilms is critical. To inhibit biofilm growth, three basic strategies have been studied such as inhibition of the initial attachment of bacteria to biotic and/or abiotic surfaces, disruption of targets of biofilm during the maturation process, and signal interference approach or Quorum Quenching (QQ). ${ }^{72}$ Inhibition of initial biofilm attachment is mediated by alteration of chemical and changing physical properties of biomaterials whereas removal of biofilms is done through matrix-degrading enzymes, surfactants, physical forces and amino acids, free fatty acids, and nitric oxide donors. Moreover, Biofilm inhibition by quorum quenching strategy is carried out through degradation of QS signals, antagonizing signaling molecules, inhibition of signal synthesis, signal transduction, and signal transport. ${ }^{28,72}$

Biofilms provided disinfection resistance at doses that wiped out planktonic populations. As a result, biofilm isolates of A. baumannii are more resistant to disinfectants than planktonic bacteria. ${ }^{73}$ A positively charged bispyridinamine exhibiting antibacterial action against plaqueproducing bacteria called Octenidine dihydrochloride $(\mathrm{OH})$ is effective for the inactivation of A. baumannii biofilms on all three matrices, such as stainless steel, polystyrene, and urinary catheters, in the presence and absence of serum protein. ${ }^{74}$ Additionally, cinnamaldehyde possesses high antibiofilm capabilities, implying that it could be used to treat biofilm-related clinical problems produced by $A$. baumannii. $^{75}$

Both growth and biofilm formation is inhibited by prebiotic metabolites such as riboflavin, raffinose, citrate, inulin, trehalose, and sorbitol. As a result, prebiotics had significant anti-biofilm efficacy against the biofilm of A. baumannii. The ability of $A$. baumannii to produce 
biofilms is reduced by $75 \% \pm 6.5 \%$ in the presence of Lactobacillus rhamnosus with inulin. ${ }^{76}$ Moreover, phage therapy such as phage $\Phi$ AB6 and its Tail Fiber (TF) protein prevents and degrades the biofilm of multidrugresistant $A$. baumannii by $78 \%$ and $62 \%$ with their respective order. $^{77}$

\section{Conclusion \& Recommendation}

Acinetobacter baumannii is an ESKAPE pathogen placed under the first in the critical priority list of pathogens for novel antibiotics. ${ }^{3}$ It is responsible for epidemics of Acinetobacter-associated nosocomial infections such as community and hospital-acquired pneumonia, central nervous system infection or meningitis, bacteremia, endocarditis, skin and soft tissue infections, wound, burn, and UTI. The biofilm formation ability of $A$. baumannii on the biotic and abiotic surface is the most essential feature contributing to chronic and persistent infections, antimicrobial resistance, and strong survival in the hospital environment. Hence, multi-drug resistance (MDR) of A. baumannii becomes a serious public health concern associated with modifications of target sites, multidrug efflux pumps, permeability defects, and enzymatic degradation of drugs named $\beta$-lactamases and aminoglycosidemodifying enzymes. Due to its capacity to develop resistance to all currently known antibiotics, A. baumannii has been designated as a "red-alert" human pathogen.

Biofilm formation is most common in A. baumannii clinical isolates from immunocompromised patients in intensive care units. Biofilm promotes A. baumannii adherence and long-term survival. So, it is an important factor in the pathogenesis and it makes the treatment options complex. The formation of A. baumannii biofilms is influenced by several biological and environmental variables. The primary determinant of A. baumannii biofilm development is glucose. ${ }^{29}$ Although evidence suggests a link between biofilm formation and multiple drug resistance in $A$. baumannii, imipenem remains the drug of choice for multi-drug resistant Acinetobacter infections. The high incidence of $A$. baumannii infections linked to medical devices is caused by biofilms, making treatment and control of infection very problematic. Therefore, health care workers (HCWs) should focus on infection prevention and control (IPC) measures or activities to control device mediated $A$. baumannii biofilm-related infections and they should be strongly selective in the utilization of therapeutics in combination with antibiofilms. Moreover, researchers shall undergo further studies which explore the exact magnitude, pathogenesis, aggravating factors, as well as mechanisms involved in biofilm formation of $A$. baumannii and the most possible therapeutics options.

\section{Author Contributions}

All authors contributed significantly to the paper's conception and design, data collection, and writing the paper; agreed to submit the manuscript to the current journal; granted final approval of the version to be published.

\section{Funding}

No funding sources.

\section{Disclosure}

The authors declare that they have no conflicts of interest.

\section{References}

1. Evans B, Hamouda A, Amyes SGB. The rise of carbapenem-resistant Acinetobacter baumannii. Curr Pharm Des. 2013;19(2):223-238. doi:10.2174/138161213804070285

2. Lin MF, Lan CY. Antimicrobial resistance in Acinetobacter baumannii: from bench to bedside. World J Clin Cases. 2014;2(12):787-814. doi:10.12998/wjcc.v2.i12.787

3. Mulani MS, Kamble EE, Kumkar SN, Tawre MS, Pardesi KR. Tawre MS and pardesi KR. emerging strategies to combat ESKAPE pathogens in the era of antimicrobial resistance: a review. Front Microbiol. 2019;10(539):1-24. doi:10.3389/fmicb.2019.00539

4. Joly-Guillou ML. Clinical impact and pathogenicity of Acinetobacter. Clin Microbiol Infect. 2005;11(11):868-873. doi:10.1111/j.14690691.2005.01227.x

5. Pour NK, Dusane DH, Dhakephalkar PK, Zamin FR, Zinjarde SS, Chopade BA. Biofilm formation by Acinetobacter baumannii strains isolated from urinary tract infection and urinary catheters. FEMS Immunol Med Microbiol. 2011;62(3):328-338. doi:10.1111/j.1574695X.2011.00818.X

6. Liu A, Du W, Xie J, et al. Role of immunodeficiency in Acinetobacter baumannii associated pneumonia in mice. Chin Med J. 2020;133(18):2161-2169. doi:10.1097/CM9.0000000000001027

7. McConnell MJ, Actis L, Pachón J. Acinetobacter baumannii: human infections, factors contributing to pathogenesis and animal models. FEMS Microbiol Rev. 2013;37(2):130-155. doi:10.1111/j.15746976.2012.00344.x

8. Falagas ME, Rafailidis PI. Attributable mortality of Acinetobacter baumannii: no longer a controversial issue. Critl Care. 2007;11 (3):1-3. doi:10.1186/cc5911

9. Antunes LCS, Visca P, Towner KJ. Acinetobacter baumannii: evolution of a global pathogen. Pathog Dis. 2014;71(3):292-301. doi:10.1111/2049-632X.12125

10. Antunes LCS, Imperi F, Carattoli A, Visca P. Deciphering the multifactorial nature of Acinetobacter baumannii pathogenicity. PLoS One. 2011;6(8):1-10. doi:10.1371/journal.pone.0022674

11. Gordon NC, Wareham DW. Multidrug-resistant Acinetobacter baumannii: mechanisms of virulence and resistance. Int $J$ Antimicrob Agents. 2010;35(3):219-226. doi:10.1016/j.ijantimicag.2009.10.024

12. Kim YJ, Kim SI, Kim YR, et al. Carbapenem-resistant Acinetobacter baumannii: diversity of resistant mechanisms and risk factors for infection. Epidemiol Infect. 2012;140(1):137-145. doi:10.1017/ S0950268811000744 
13. Yang $\mathrm{CH}, \mathrm{Su} \mathrm{PW}$, Moi SH, Chuang LY. Biofilm formation in Acinetobacter baumannii: genotype-phenotype correlation. Molecules. 2019;24(10):1-12.

14. Roy R, Tiwari M, Donelli G, Tiwari V. Strategies for combating bacterial biofilms: a focus on anti-biofilm agents and their mechanisms of action. Virulence. 2018;9(1):522-554. doi:10.1080/ 21505594.2017.1313372

15. Asfaw T. Biofilm formation by Enterococcus Faecalis and Enterococcus Faecium: review. Int J Res Stud Biosci. 2019;7(4). doi:10.20431/2349-0365.0704002

16. Runci F, Bonchi C, Frangipani E, Visaggio D, Visca P. Acinetobacter baumannii biofilm formation in human serum and disruption by gallium. Antimicrob Agents Chemother. 2017;61(1):1-8. doi:10.1128/AAC.01563-16

17. Dijkshoorn L, Nemec A, Seifert H. An increasing threat in hospitals: multidrug-resistant Acinetobacter baumannii. Nat Rev Microbiol. 2007;5(12):939-951. doi:10.1038/nrmicro1789

18. Babapour E, Haddadi A, Mirnejad R, Angaji SA, Amirmozafari N. Biofilm formation in clinical isolates of nosocomial Acinetobacter baumannii and its relationship with multidrug resistance. Asian Pac J Trop Biomed. 2016;6(6):528-533. doi:10.1016/j. apjtb.2016.04.006

19. Ebrahimi S. Efficacy of isolated bacteriophage against biofilm embedded colistin-resistant Acinetobacter baumannii. Gene Rep. 2021;22:1-11.

20. Krzyściak P, Chmielarczyk A, Pobiega M, Romaniszyn D, Wójkowska-Mach J. Acinetobacter baumannii isolated from hospital-acquired infection: biofilm production and drug susceptibility. Apmis. 2017;125(11):1017-1026. doi:10.1111/ apm. 12739

21. Rodríguez-Baño J, Martí S, Soto S, et al. Biofilm formation in Acinetobacter baumannii: associated features and clinical implications. Clin Microbiol Infect. 2008;14(3):276-278. doi:10.1111/j.1469-0691.2007.01916.x

22. Zeighami H, Valadkhani F, Shapouri R, Samadi E, Haghi F. Virulence characteristics of multidrug resistant biofilm forming Acinetobacter baumannii isolated from intensive care unit patients. BMC Infect Dis. 2019;19(1):1-9. doi:10.1186/s12879-019-4272-0

23. Bala M, Gupte S, Aggarwal P, Kaur M, Manhas A. Biofilm producing multidrug resistant Acinetobacter species from a tertiary care hospital: a therapeutic challenge. Int J Res Med Sci. 2016;4(7):3024-3026. doi:10.18203/2320-6012.ijrms20161997

24. Fajir L, Al-niaame AE, Hussein N. Detection of biofilm formation and its related with aminoglycoside resistance in Acinetobacter baumannii isolates, isolated from some Baghdad City Hospitals. Eur J Biomed Pharm Sci. 2017;4(1):84-91.

25. Rao R, Karthika R, Singh S, et al. Correlation between biofilm production and multiple drug resistance in imipenem resistant clinical isolates of Acinetobacter baumannii. Indian J Med Microbiol. 2008;26(4):333-337. doi:10.1016/S0255-0857(21)01809-0

26. Azizi O, Shahcheraghi F, Salimizand H, et al. Molecular analysis and expression of bap gene in biofilm-forming multi-drug-resistant Acinetobacter baumannii. Rep Biochem Mol Biol. 2016;5(1):62-72.

27. Blanco N, Harris AD, Rock C, et al. Risk factors and outcomes associated with multidrug-resistant Acinetobacter baumannii upon intensive care unit admission. Antimicrob Agents Chemother. 2018;62(1):1-7. doi:10.1128/AAC.01631-17

28. Elkheloui R, Laktib A, Mimouni R, et al. Acinetobacter baumannii biofilm: intervening factors, persistence, drug resistance, and strategies of treatment. Mediterr J Infect Microb Antimicrob. 2020;9 (7):1-12.

29. Eze EC, Chenia HY, El Zowalaty ME. Acinetobacter baumannii biofilms: effects of physicochemical factors, virulence, antibiotic resistance determinants, gene regulation, and future antimicrobial treatments. Infect Drug Resist. 2018;11:2277-2299. doi:10.2147/ IDR.S169894
30. Jamal M, Tasneem U, Hussein TAS. Bacterial biofilm: its composition, formation and role in human infections. Research \& reviews. J Microbiol Biotechnol. 2015;4(3):1-14.

31. Garrett TR, Bhakoo M, Zhang Z. Bacterial adhesion and biofilms on surfaces. Prog Nat Sci. 2008;18(9):1049-1056. doi:10.1016/j. pnsc.2008.04.001

32. Jamal M, Ahmad W, Andleeb S, et al. Bacterial biofilm and associated infections. J Chin Med Assoc. 2018;81(1):7-11. doi:10.1016/j. jcma.2017.07.012

33. Stewart PS, Franklin MJ. Physiological heterogeneity in biofilms. Nat Rev Microbiol. 2008;6(3):199-210. doi:10.1038/nrmicro1838

34. Hannan S, Ready D, Jasni AS, Rogers M, Pratten J, Roberts AP. Transfer of antibiotic resistance by transformation with eDNA within oral biofilms. FEMS Immunol Med Microbiol. 2010;59(3):345-349. doi:10.1111/j.1574-695X.2010.00661.x

35. Parsek MR, Singh PK. Bacterial biofilms: an emerging link to disease pathogenesis. Annu Rev Microbiol. 2003;57:677-701. doi:10.1146/ annurev.micro.57.030502.090720

36. Bekele ST, Abay GK, Gelaw B TB. Bacterial Biofilms; Links to Pathogenesis and Résistance Mechanism. 2018;1-25. doi:10.20944/ preprints201807.0598.v1

37. Greene C, Vadlamudi G, Newton D, Foxman B, Xi C. The influence of biofilm formation and multidrug resistance on environmental survival of clinical and environmental isolates of Acinetobacter baumannii. Am J Infect Control. 2016;44(5):65-71. doi:10.1016/j. ajic.2015.12.012

38. Richards AM, Abu Kwaik Y, Lamont RJ. Code blue: Acinetobacter baumannii, a nosocomial pathogen with a role in the oral cavity. Mol Oral Microbiol. 2015;30(1):2-15. doi:10.1111/omi.12072

39. Cerqueira GM, Peleg AY. Insights into Acinetobacter baumannii pathogenicity. IUBMB Life. 2011;63(12):1055-1060. doi:10.1002/iub.533

40. Sato Y, Unno Y, Kawakami S, Ubagai T, Ono Y. Virulence characteristics of Acinetobacter baumannii clinical isolates vary with the expression levels of omps. J Med Microbiol. 2017;66(2):203-212. doi:10.1099/jmm.0.000394

41. Gaddy JA, Actis LA. Regulation of Acinetobacter baumannii biofilm formation Jennifer. Future Microbiol. 2009;4(1):273-278. doi: $10.2217 / \mathrm{fmb} .09 .5$

42. Gaddy JA, Tomaras AP, Actis LA. The Acinetobacter baumannii 19606 ompa protein plays a role in biofilm formation on abiotic surfaces and in the interaction of this pathogen with eukaryotic cells. Infect Immun. 2009;77(8):3150-3160. doi:10.1128/IAI.00096-09

43. Choi CH, Lee JS, Lee YC, Park TI, Lee JC. Acinetobacter baumannii invades epithelial cells and outer membrane protein A mediates interactions with epithelial cells. BMC Microbiol. 2008;8(1):1-11. doi:10.1186/1471-2180-8-216

44. Choi CH, Lee EY, Lee YC, et al. Outer membrane protein 38 of Acinetobacter baumannii localizes to the mitochondria and induces apoptosis of epithelial cells. Cell Microbiol. 2005;7(8):1127-1138. doi:10.1111/j.1462-5822.2005.00538.x

45. Choi CH, Hyun SH, Lee JY, et al. Acinetobacter baumannii outer membrane protein A targets the nucleus and induces cytotoxicity. Cell Microbiol. 2008;10(2):309-319.

46. Loehfelm TW, Luke NR, Campagnari AA. Identification and characterization of an Acinetobacter baumannii biofilm-associated protein. J Bacteriol. 2008;190(3):1036-1044. doi:10.1128/JB.01416-07

47. Boll JM, Tucker AT, Klein DR, et al. Reinforcing lipid A acylation on the cell surface of Acinetobacter baumannii promotes cationic antimicrobial peptide resistance and desiccation survival. mBio. 2015;6 (3):1-11. doi:10.1128/mBio.00478-15

48. Luke NR, Sauberan SL, Russo TA, et al. Identification and characterization of a glycosyltransferase involved in Acinetobacter baumannii lipopolysaccharide core biosynthesis identification and characterization of a glycosyltransferase involved in Acinetobacter baumannii lipopolysaccharide core. Infect Immun. 2010;78(5):2017-2023. doi:10.1128/IAI.00016-10 
49. Russo TA, Macdonald U, Beanan JM, et al. Penicillin-binding protein $7 / 8$ contributes to the survival of Acinetobacter baumannii in vitro and in vivo. Infect Immun. 2009;199:513-521.

50. Jin JS, Kwon S, Moon DC, et al. Acinetobacter baumannii secretes cytotoxic outer membrane protein A via outer membrane vesicles. PLoS One. 2011;6(2):1-9. doi:10.1371/journal.pone.0017027

51. Rumbo C, Ferna E, Merino M, et al. Horizontal transfer of the OXA-24 carbapenemase gene via outer membrane vesicles: a new mechanism of dissemination of carbapenem resistance genes in Acinetobacter baumannii. Antimicrob Agents Chemother. 2011;55 (7):3084-3090. doi:10.1128/AAC.00929-10

52. Jacobs AC, Hood I, Boyd KL, et al. Inactivation of phospholipase $\mathrm{D}$ diminishes Acinetobacter baumannii pathogenesis. Infect Immun. 2010;78(5):1952-1962. doi:10.1128/IAI.00889-09

53. Gaddy JA, Arivett BA, Mcconnell MJ, López-rojas R, Pachón J, Actis LA. Role of acinetobactin-mediated iron acquisition functions in the interaction of Acinetobacter baumannii strain ATCC 19606 $\mathrm{T}$ with human lung epithelial cells, Galleria mellonella caterpillars, and mice. Infect Immun. 2012;80:1015-1024. doi:10.1128/ IAI.06279-11

54. Mihara K, Tanabe T, Yamakawa Y, et al. Identification and transcriptional organization of a gene cluster involved in biosynthesis and transport of acinetobactin, a siderophore produced by Acinetobacter baumannii ATCC 19606 T. Microbiology. 2004;150:2587-2597. doi:10.1099/mic.0.27141-0

55. Geisinger E, Isberg RR, Weiss D. Antibiotic modulation of capsular exopolysaccharide and virulence in Acinetobacter baumannii. PLoS Pathog. 2015;11(2):1-28. doi:10.1371/journal.ppat.1004691

56. Fiester SE, Arivett BA, Schmidt RE, et al. Iron-Regulated phospholipase $\mathrm{C}$ activity contributes to the cytolytic activity and virulence of Acinetobacter baumannii. PLoS One. 2016;11(11):1-26. doi:10.1371/ journal.pone. 0167068

57. Camarena L, Bruno V, Euskirchen G, Poggio S, Snyder M. Molecular mechanisms of ethanol-induced pathogenesis revealed by RNA-sequencing. PLoS Pathog. 2010;6(4):1-15. doi:10.1371/journal.ppat. 1000834

58. Choi AHK, Slamti L, Avci FY, Pier GB, Maira-Litran T. The pgaABCD locus of Acinetobacter baumannii encodes the production of Poly- $\beta-1-6-\mathrm{N}$-acetylglucosamine, which is critical for biofilm formation. $J$ Bacteriol. 2009;191(19):5953-5963. doi:10.1128/ JB.00647-09

59. Tomaras AP, Flagler MJ, Dorsey CW, Gaddy JA, Actis LA. Characterization of a two-component regulatory system from Acinetobacter baumannii that controls biofilm formation and cellular morphology. Microbiology. 2008;154:3398-3409. doi:10.1099/ mic. 0.2008/019471-0

60. Niu C, Clemmer KM, Bonomo RA, Rather PN. Isolation and characterization of an autoinducer synthase from Acinetobacter baumannii. J Bacteriol. 2008;190(9):3386-3392. doi:10.1128/ JB.01929-07

61. Tomaras AP, Dorsey CW, Edelmann RE, Actis LA. Attachment to and biofilm formation on abiotic surfaces by Acinetobacter baumannii: involvement of a novel chaperone-usher pili assembly system. Microbiology. 2003;149:3473-3484. doi:10.1099/mic.0.26541-0
62. Amala Reena AA, Subramaniyan A, Kanungo R. Biofilm formation as a virulence factor of Acinetobacter baumannii: an emerging pathogen in critical care units. J Curr Res Sci Med. 2017;3(2):74. doi: $10.4103 /$ jcrsm.jcrsm_66_17

63. Princy Choudhary SS. Microbial biofilms. Encycl Microbiol. 2019;1-14. doi:10.5772/intechopen.90790

64. Toyofuku M, Inaba T, Kiyokawa T, Obana N, Yawata Y, Nomura N. Environmental factors that shape biofilm formation. Biosci Biotechnol Biochem. 2016;80(1):7-12. doi:10.1080/ 09168451.2015.1058701

65. Sindhu S. Clinical significance, antibiotic resistance and biofilm formation of Acinetobacter baumannii. Rev Clin Microbiol. 2018;7(4):1-2.

66. Badave GK, Dhananjay K. Biofilm producing multidrug resistant Acinetobacter baumannii: an emerging challenge. JCDR. 2015;9 (1):9-11.

67. Rodrigues Perez LR. Acinetobacter baumannii displays inverse relationship between meropenem resistance and biofilm production. JChemother. 2015;27(1):13-15. doi:10.1179/1973947813Y.0000000159

68. Madanan A, Kumari S, Routray A, Yadav D. Imipenem resistance and biofilm production in Acinetobacter. Drug Invent Today. 2013;5 (3):256-258. doi:10.1016/j.dit.2013.04.005

69. Høiby N, Ciofu O, Johansen HK, et al. The clinical impact of bacterial biofilms. Int J Oral Sci. 2011;3:55-65. doi:10.4248/ IJOS11026

70. Diaz PM. Impact of biofilm infection and its treatment impact of biofilm infection and its treatment. Int $J$ Adv Microbiol Microbiol Res. 2017;1(1):7-13.

71. Wu H, Moser C, Wang H, Høiby N, Song Z. Strategies for combating bacterial biofilm infections. Int J Oral Sci. 2014;7:1-7. doi:10.1038/ ijos. 2014.65

72. Subhadra B, Kim DH, Woo K, Surendran S, Choi CH. Control of biofilm formation in healthcare: recent advances exploiting quorum-sensing interference strategies and multidrug efflux pump inhibitors. Materials. 2018;11(9):1-20. doi:10.3390/ma11091676

73. Ivanković T, Goić-Barišić I, Hrenović J. Reduced susceptibility to disinfectants of Acinetobacter baumannii biofilms on glass and ceramic. Arh Hig Rada Toksikol. 2017;68(2):99-108. doi:10.1515/ aiht-2017-68-2946

74. Narayanan A, Nair MS, Karumathil DP, Baskaran SA, Venkitanarayanan K, Amalaradjou MAR. Inactivation of Acinetobacter baumannii biofilms on polystyrene, stainless steel, and urinary catheters by octenidine dihydrochloride. Front Microbiol. 2016;7:1-9. doi:10.3389/fmicb.2016.00847

75. Mohamed SH, Salem D, Azmy M, Fam NS. Antibacterial and antibiofilm activity of cinnamaldehyde against carbapenem-resistant Acinetobacter baumannii in Egypt: in vitro study. J Appl Pharm Sci. 2018;8(11):151-156.

76. Laal-Kargar N, Dolatabadi S, Mohtashami M. Antibacterial and antibiofilm effects of synbiotics against multidrug-resistant bacteria: Acinetobacter baumannii and Enterococcus faecalis; 2020. 1-26.

77. Shahed-Al-Mahmud MD, Lin NT. Prevention and degradation of Acinetobacter baumannii biofilm formation by phage $\phi \mathrm{AB} 6$ and its tail fiber protein. Virol Mycol. 2018;07:36.
Infection and Drug Resistance

\section{Publish your work in this journal}

Infection and Drug Resistance is an international, peer-reviewed openaccess journal that focuses on the optimal treatment of infection (bacterial, fungal and viral) and the development and institution of preventive strategies to minimize the development and spread of resistance. The journal is specifically concerned with the epidemiology of antibiotic resistance and the mechanisms of resistance development and diffusion in both hospitals and the community. The manuscript management system is completely online and includes a very quick and fair peerreview system, which is all easy to use. Visit http://www.dovepress.com/ testimonials.php to read real quotes from published authors. 